\title{
Exacerbation of Psoriasis Confirmed by Drug Challenge Test of Lithium Carbonate
}

\author{
Tomotaka Mabuchi, Hanako Yamaoka \\ Department of Dermatology, Tokai University School of Medicine, 143 Shimokasuya, Isehara, \\ Kanagawa, Japan \\ mabuchi@is.icc.u-tokai.ac.jp
}

Keywords: psoriasis, bipolar affective disorders, lithium carbonate

\section{INTRODUCTION}

Psoriasis is a common, inflammatory, and proliferative disease of the skin. The exact cause of psoriasis is unclear, but it is thought to be a multifactorial disease triggered by both genetic and environmental factors such as trauma, infection, alcohol, smoking, stress, and drugs. It is established that lithium carbonate induce and/or exacerbate psoriasis [1]. Herein, we report a case of exacerbation of psoriasis which was confirmed by drug challenge test of lithium carbonate.

\section{CASE Report}

A 58-year-old Japanese male visited the outpatient clinic of our hospital because of scaly erythema on his trunk and extremities over the past three years. On the initial dermatological examination, we noticed various sizes of round, sharply bordered and itchy erythematous plaques with silvery scale involving the head, trunk, and extremities (Figure 1). The initial hematology tests, biochemistry tests and urinalysis revealed normal. A skin biopsy was performed from the plaque on his left upper arm. Histopathological examination showed parakeratosis of epidermis with elongated rete ridge. Aggregations of neutrophils are focally seen in the subcorneal layer. Mild lymphocytic infiltration is noted around vessels in the upper dermis. Together, these clinical and histopathological findings led to the diagnosis of psoriasis vulgaris. The skin lesions did not respond to topical corticosteroid and vitamin $\mathrm{D}_{3}$ analogue.

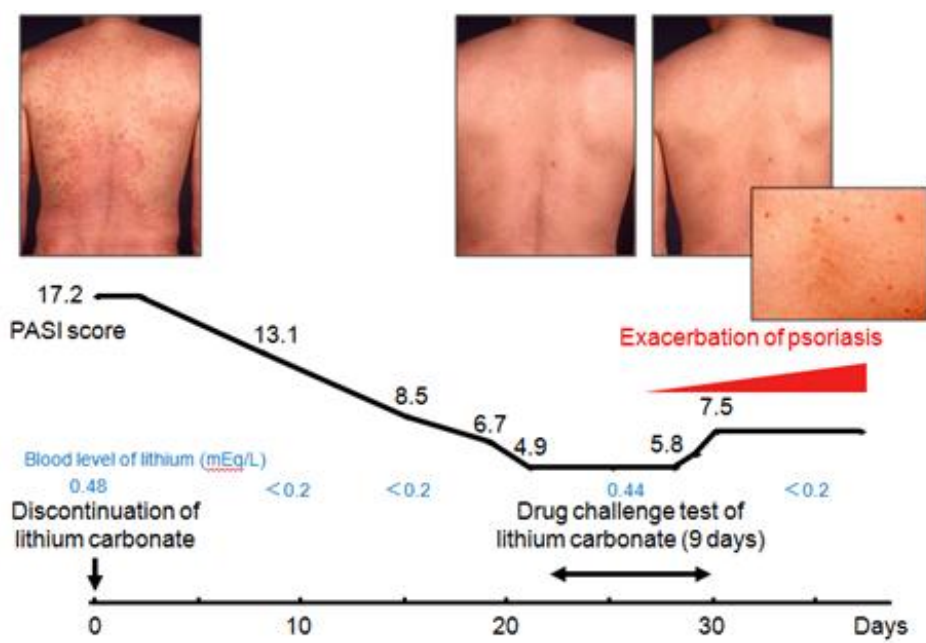

Fig1. Course of clinical features, PASI score, and blood level of lithium during drug challenge test of lithium carbonate

The patient had bipolar affective disorders and had been treated with multiple medications including $600 \mathrm{mg} /$ day of lithium carbonate for approximately ten months. After initiation of lithium carbonate treatment, his psoriatic skin lesions had worsen, therefore, we had suspected this to be the cause of exacerbation, and stopped the lithium carbonate treatment after consultation with his psychiatrist. 
Psoriatic skin lesions improved during the next three weeks of discontinuance of lithium carbonate treatment; Psoriasis area and severity index (PASI) score was decreased from 17.2 to 4.9 (Figure 1). Drug challenge test of $600 \mathrm{mg} /$ day of lithium carbonate was undergone after obtaining informed consent from the patient. Treatment with topical corticosteroid and vitamin $\mathrm{D}_{3}$ analogue had been continued through the drug challenge test of lithium carbonate. Erythema, infiltration, and pruritus had exacerbated with new psoriatic skin eruptions at day 9 of drug challenge test of lithium carbonate. During the course of another ten days, at day 19, PASI score rose to 7.5 (Figure 1). Neither patch test nor drug lymphocyte stimulating test were positive for lithium carbonate.

\section{DisCuSSION}

Lithium carbonate is the most widely used long-term treatment for bipolar affective disorders [2]. Exacerbation and provocation of psoriasis by lithium carbonate were first reported in 1972 [3] and 1976 [4] , respectively [1]. The mechanism of action of lithium carbonate in exacerbation or provocation of psoriasis is still unclear, but lithium carbonate could affect psoriasis in several ways. One of which may be through the inositol metabolic pathway [2]. Decrease in inositol which results from lithium carbonate causes low intracellular levels of calcium, leading to lack of differentiation and increased proliferation of keratinocytes, enhanced chemotaxis, and phagocytic activity of polymorphonuclear leukocytes [1]. A small, randomized, placebo-controlled trial demonstrated that the inositol supplements had a significantly beneficial effect on the psoriatic patients taking lithium carbonate [2]. Lithium carbonate also affects cytokine pathways. Many cytokines are potentially involved in the development and maintenance of psoriasis. Interleukin (IL)-17 and IL-23 as well as tumor necrosis factor (TNF) $\alpha$ are key cytokines in the pathogenesis of psoriasis [5]. Both anti-IL-17 antibodies, anti-IL-12/23p40 antibody, and TNF $\alpha$ inhibitors are very efficacious for psoriasis. It is reported that lithium carbonate activates the production of cytokines that promote inflammatory processes such as IL-1 $\beta$, IL-2, IL-6, IL-17, and TNF $\alpha$ [6]. Both IL-1 $\beta$ and IL-6 are key cytokines for the development of human Th17 cells from naïve T cells. Furthermore, in vitro study reported that lithium carbonate regulates keratinocyte proliferation via glycogen synthase kinase 3 and nuclear factor of activated T cells 2 [7].

There are many studies which reported the association of psoriasis with depression. A systematic review and meta-analysis study reported that more than $10 \%$ of psoriatic patients suffer from clinical depression and twice as many have depressive symptoms [8]. Therefore, there are always possibilities that our psoriatic patients had taken lithium carbonate in the past, or would take lithium carbonate in the future, as the first line treatment for depression.

\section{Conclusion}

In conclusion, increased edification of exacerbation and provocation of psoriasis by lithium carbonate is still important for all dermatologists, psychiatrists, and pharmacists.

\section{REFERENCES}

[1] Basavaraj, K. H., N. M. Ashok, R. Rashmi, and T. K. Praveen. 2010. The role of drugs in the induction and/or exacerbation of psoriasis. Int. J. Dermatol. 49: 1351-1361.

[2] Allan, S. J., G. M. Kavanagh, R. M. Herd, and J. A. Savin. 2004. The effect of inositol supplements on the psoriasis of patients taking lithium: a randomized, placebo-controlled trial. Br. J. Dermatol. 150: 966-969.

[3] Carter, T. N. 1972. The relationship of lithium carbonate to psoriasis. Psychosomatics 13: 325-327.

[4] Bakker, J. B. and L. Pepplinkhuizen. 1976. More about the relationship of lithium to psoriasis. Psychosomatics 17: 143-146.

[5] Lowes, M. A., C. B. Russell, D. A. Martin, J. E. Towne, and J. G. Krueger. 2013. The IL-23/T17 pathogenic axis in psoriasis is amplified by keratinocyte responses. Trends Immunol. 34: 174-181.

[6] Petersein, C., U. Sack, R. Mergl, J. Schonherr, F. M. Schmidt, N. Lichtblau, K. C. Kirkby, K. Bauer, and H. Himmerich. 2015. Impact of lithium alone and in combination with antidepressants on cytokine production in vitro. J. Neural Transm. (Vienna) 122: 109-122.

[7] Hampton, P. J., R. Jans, R. J. Flockhart, G. Parker, and N. J. Reynolds. 2012. Lithium regulates keratinocyte proliferation via glycogen synthase kinase 3 and NFAT2 (nuclear factor of activated T cells 2). J. Cell. Physiol. 227: 1529-1537.

[8] Dowlatshahi, E. A., M. Wakkee, L. R. Arends, and T. Nijsten. 2014. The prevalence and odds of depressive symptoms and clinical depression in psoriasis patients: a systematic review and meta-analysis. J. Invest. Dermatol. 134: 1542-1551. 